\title{
English adaptation in Mandarin A-not-A constructions
}

\author{
Minqi Liu*
}

\begin{abstract}
A-not-A refers to a Mandarin reduplication construction where the underlying form /RED-pu-A/ contains a reduplication of the first syllable in $A$. In this study I investigate the kinds of adaptations that occur when an English word serves as the base $A$ in code-switching speech. Since the complex onsets and most codas allowed in English are illegal in Mandarin syllables, the reduplicated part is expected to adapt to Mandarin phonotactics to some degree. I ran a production experiment where 20 native Mandarin-speakers were asked to produce A-not-A constructions with 55 mono- and multi-syllabic English words. Results from the experiment showed varied adaptation methods in syllable structure and tones. To model the results, I used the Maximum Entropy Harmonic Grammar (MaxEnt) with weighted constraints on syllable structure markedness and base-reduplicant faithfulness.
\end{abstract}

Keywords. reduplication; Mandarin-English code-switching; phonotactics; sonority; tone; maximum entropy

1. Introduction. Code-switching to English in colloquial speech is common for Mandarinspeakers, especially younger generations with certain amount of English-exposure. This paper investigates an intriguing phenomenon observed among native Mandarin-speaking international students in Los Angeles, how they reduplicate English words in a Mandarin reduplication construction, namely the A-not-A construction.

An A-not-A construction in Mandarin contains two syntactic copies of $A$ ( $A$ can be an adjective or a verb) that are intervened by a negative adverb $b u$ [pu] 'not', hence the name. It is a reduplication construction by the virtue that the first $A$ is phonologically realized as a copy of the first syllable ${ }^{1}$ of the second $A$ element. Therefore, the first $A$ is a reduplicant and the second $A$ serves as its base. (1) is an example with a Mandarin word xinxian $\left[\operatorname{cin}^{55} . \operatorname{cien}^{55}\right]$ :

$$
\begin{aligned}
& \sin ^{55} \mathrm{pu}^{51} \operatorname{cin}^{55} \cdot \operatorname{cien}^{55} \\
& \sigma_{\text {RED }} \text { not fresh } \\
& \text { 'fresh or not' }
\end{aligned}
$$

When Mandarin-speakers code-switch to English while producing this construction and introduce English words as the base, the base part of this reduplication is always faithful to its input but the reduplicant adapts to Mandarin phonotactics to some degree:

$$
\begin{aligned}
& \text { (2) } \mathrm{pu}^{35} \text { fre } \int \\
& \sigma_{\mathrm{RED}} \text { not fresh } \\
& \text { 'fresh or not' }
\end{aligned}
$$

* Many thanks to the audience at the 93rd Annual Meeting of LSA and members of the UCLA phonology seminar. Thanks also to Zhongshi Xu for his helpful input, my RAs Weikang Xiong and Mengxin Zhu for their contribution in subject recruitment and data collection, and Maddy Booth for her beautiful voice. All errors remain mine. Author: Minqi Liu, University of California, Los Angeles (liuminqi@ucla.edu).

${ }^{1}$ An alternative A-not-A reduplication grammar in Mandarin is to reduplicate the whole word regardless of the syllable numbers, which is beyond this paper's discussion. 
As seen in (2), the coda [ $\left.\int\right]$ is deleted in the reduplicant due to the restriction in Mandarin that only allows two consonants in coda position, [n] and [y]. But the complex onset [fr] of the reduplicant, which is also impossible in Mandarin, gets preserved in the onset position.

This paper will investigate the adaptation patterns of English words reduplicated in A-notA constructions with a production experiment, aiming to answer the following questions: How will English syllables adapt to Mandarin constraints when reduplicated in Mandarin A-notA constructions and what does it tell us about Mandarin? Section 2 describes the production experiment and section 3 demonstrates the adaptations of English in this construction with respect to onset, coda, and tonal adaptations. Section 4 models the results of the experiment in section 2 by using the Maximum Entropy Harmonic Grammar (MaxEnt; Goldwater \& Johnson 2003) with weighted constraints on syllable structure markedness and base-reduplicant faithfulness. Section 5 summarizes this paper.

\section{Production Experiment.}

2.1 Subjects And TeSt EnVIROnMEnT. 20 native Mandarin-speakers were recruited. They were undergraduate students at the University of California, Los Angeles participating for course credits. They were individually tested on a computer with internal speakers and microphones in quiet rooms in the Department of Linguistics at UCLA. Three subjects were excluded because their reduplication grammar for all the English words shows an alternative Anot-A forming rule in Mandarin which is beyond this paper.

2.2 MATERiAls AND PROCEDURE. This experiment was built on the platform AppsoBab$b^{2} e^{2}$ for online tests. During the test, participants clicked a button on the screen to hear a word without seeing it, and then produce its A-not-A form based on their intuition.

In the training session, participants heard and produced the A-not-A forms for 3 Mandarin disyllabic words pre-recorded by a native Mandarin-speaker. 55 English words were tested. All the tested English words were pre-recorded by a native North American English-speaker and were semantically compatible with the Mandarin A-not-A constructions. There were 43 monosyllabic words varying with onset and coda conditions: (i) Onset-17 words with simple onsets and 26 with complex onsets; (ii) Coda-10 words with no coda, 5 with codas that are legal in Mandarin (namely [n] and [n]), and 28 with different codas that are not in Mandarin inventory. There were also 12 multi-syllabic English words, half with stress on the first syllable and half on other syllables.

2.3 Data collection. Participants' production was recorded by the online platform. All data points were analyzed by well-trained experimenters. In total, 26 misheard items were excluded. The results of this experiment are presented in sections 3.1 and 3.2.

3. English Adaptation in Mandarin A-not-A Constructions. This section demonstrates the results from the experiment by the following order: onset adaptations, coda adaptations, tonal adaptations, and other interesting phenomena during the production. Based on these results, I propose that the segmental adaptations reflect the effects of sonority and the tone of the reduplicant is faithful to the first tone in the base.

\footnotetext{
${ }^{2}$ Website: http://www.appsobabble.com
} 
3.1 AdAPTATION OF ONSETS. English simple onsets are always faithful to its input, the onset of the base, even when the onset is not in the Mandarin inventory, such as [ $]$.

$$
\begin{aligned}
& \int o v \mathrm{pu}^{35} \text { fow } \\
& \sigma_{\text {RED }} \text { not show } \\
& \text { 'show or not' }
\end{aligned}
$$

The faithfulness of the simple onsets is attested in all the data collected, although the base itself might not always faithful to its English input form, affected by the L2-speaker's English fluency. For example, a voiced onset [b] might lose its voicedness during Mandarin-speakers' production due to reasons independent of reduplication. In that case, even the base onset [p] is not faithful to its English input [b], the onset of the reduplicant [p] is still counted as faithful to the onset of the base.

As for complex onsets in English, two main strategies were observed during the test: faithful production and vowel insertion. An item is counted as faithful production if all the segments in a complex onset are preserved in the onset of the reduplicant. (2) is an example of faithfully producing the complex onset [fr]. The other strategy only reduplicates the the first consonant in the complex onset and inserts a vowel after it to form a syllable. Table 1 shows

\begin{tabular}{|c|c|c|c|c|}
\hline onset type & base & \multicolumn{3}{|c|}{$\sigma_{\mathrm{RED}}$} \\
\hline$/ \mathrm{sTL} /$ & splash : /splæ J/ & splæ & 7 & S.I \\
\hline /sT/ & spit : /spit/ & spI & / & SI \\
\hline$/ \mathrm{Cl} /$ & clean : /klin/ & klin & / & kə \\
\hline$/ \mathrm{Cr} /$ & fresh : /fref/ & fre & I & $\mathrm{fu}$ \\
\hline
\end{tabular}
these two strategies with different types of complex onsets tested in the experiment $(\mathrm{C}=$ consonants, $\mathrm{T}=$ stops, $\mathrm{L}=$ liquids):

Table 1: Two strategies of reduplicating complex onsets

Although both strategies are applicable during the production process, they are not applied equally across all types of complex onset. For example, [fr] in fresh is more likely to be faithfully preserved than [sp] in spit, as shown in Table 2:

\begin{tabular}{lcc} 
& spit & fresh \\
\hline faithful production & $53 \%$ & $73 \%$ \\
vowel insertion & $47 \%$ & $27 \%$ \\
\hline
\end{tabular}

Table 2: Production of complex onsets in spit and fresh

The percentages of these two strategies across production of the four types of complex onsets are shown in Figure 1, according to which $/ \mathrm{Cr} /$ is the complex onset that has the most faithful production while /sTL/ is the least. I propose that consonant clusters with falling sonority, such as /sTL/ and /sT/, are more likely to split and undergo vowel insertion than clusters with rising sonority from the left edge, such as $/ \mathrm{Cl} /$ and $/ \mathrm{Cr} /$.

This effect of sonority is in line with the Sonority Sequencing Principle (SSP) that the nucleus of a syllable constitutes its sonority peak and from the nucleus toward both edges of the syllable, the sonority falls. Mandarin perfectly fulfills SSP due to its simple syllable structure 
that allows at most one onset before the nucleus and one coda after, while some of English complex onsets like /sTL/ and /sT/ creates a sonority dipping from the left edge to the nucleus, which gives rise to the splitting of $/ \mathrm{s} /$ and its following stops in the reduplication process of A-not-A constructions.

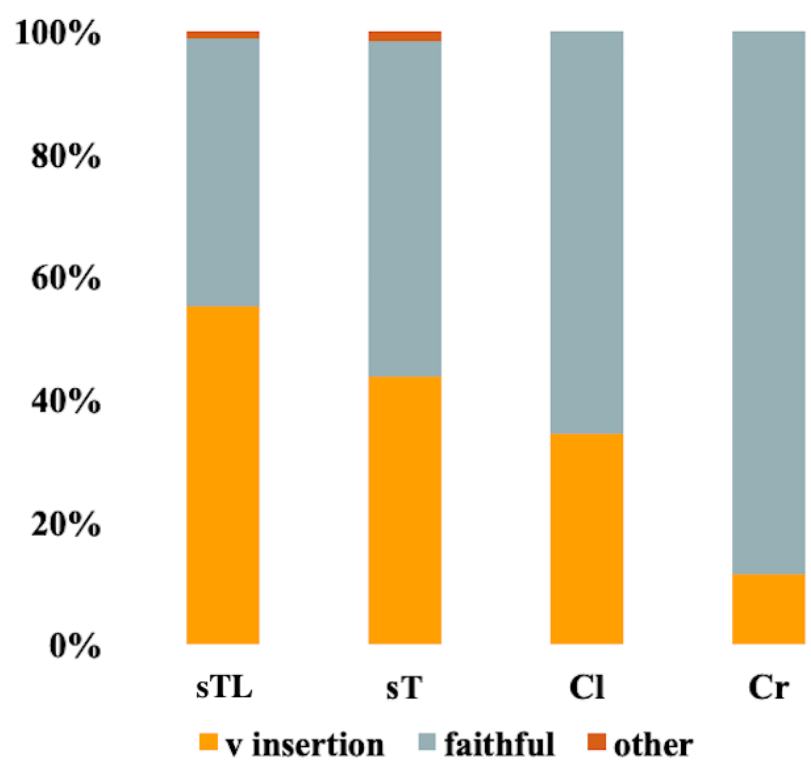

Figure 1: Adaptation of English complex onsets

Another phenomenon observed is the asymmetry between $/ \mathrm{Cl} /$ and $/ \mathrm{Cr} /$. Both $/ \mathrm{r} /$ or $/ \mathrm{l} /$ being liquids, the sonority profile of $/ \mathrm{Cl} /$ and $/ \mathrm{Cr} /$ should be approximately the same, yet $/ \mathrm{Cl} /$ is more likely to be split up than the consonant sequence $/ \mathrm{Cr} /$. I propose that this asymmetry results from the Mandarin inventory, in which $/ 1 /$ is a legitimate onset while $/ \mathrm{r} /$ is not. For this reason, inserting a syllabic boundary in $/ \mathrm{ClV} /$ between the initial $/ \mathrm{C} /$ and the following $/ \mathrm{lV} /$ sounds more natural than in the $/ \mathrm{CrV} /$ sequence because $/ \mathrm{lV} /$ is a legitimate syllable in Mandarin but $/ \mathrm{rV} /$ is not.

3.2 AdAPTATION OF CODAS. Native Mandarin phonotactics allows no other consonants in the coda position than $/ \mathrm{n} /$ and $/ \mathrm{y} /$, which means most of English codas are illegal in Mandarin. When syllables with these illegal codas are reduplicated, three adaptation strategies are observed: faithful production of the coda, coda deletion, and coda alternation. Table 3 exhibits some examples of these strategies applied to different types of codas. For instance, $[\mathrm{m}]$ in [sim] is a faithful production of the coda of $/ \operatorname{sim} /,[\mathrm{n}]$ in [sin] is an alternation, and the zero form in [si] shows the deletion.

\begin{tabular}{|c|c|c|}
\hline coda type & base & $\sigma_{\mathrm{RED}}$ \\
\hline$/ \mathrm{r} /$ or $/ 1 /$ & poor : /pur/ & pur \\
\hline & fall : /fol/ & 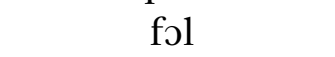 \\
\hline$/ \mathrm{m} /$ & seem $: / \operatorname{sim} /$ & $\operatorname{sim} / \sin / \operatorname{si}$ \\
\hline$[-$ son $]$ & sick : /sik/ & sIk / SI \\
\hline
\end{tabular}

Table 3: Coda adaptations 
Figure 2 illustrates different proportions of these strategies in different types of English codas in the reduplicants. One prominent finding of this experiment is that $/ \mathrm{r} /$ and $/ 1 /$, when in coda positions, are almost always preserved in the reduplicant, contrasting sharply with obstruents such as fric(atives), affr(icatives), and stops, as shown in Figure 2. Another interesting phenomenon is the alternation rate of the coda $/ \mathrm{m} /$, which constituents almost half of the case. Note that in all the alternation cases, $/ \mathrm{m} /$ becomes $/ \mathrm{n} /$ in the coda position.

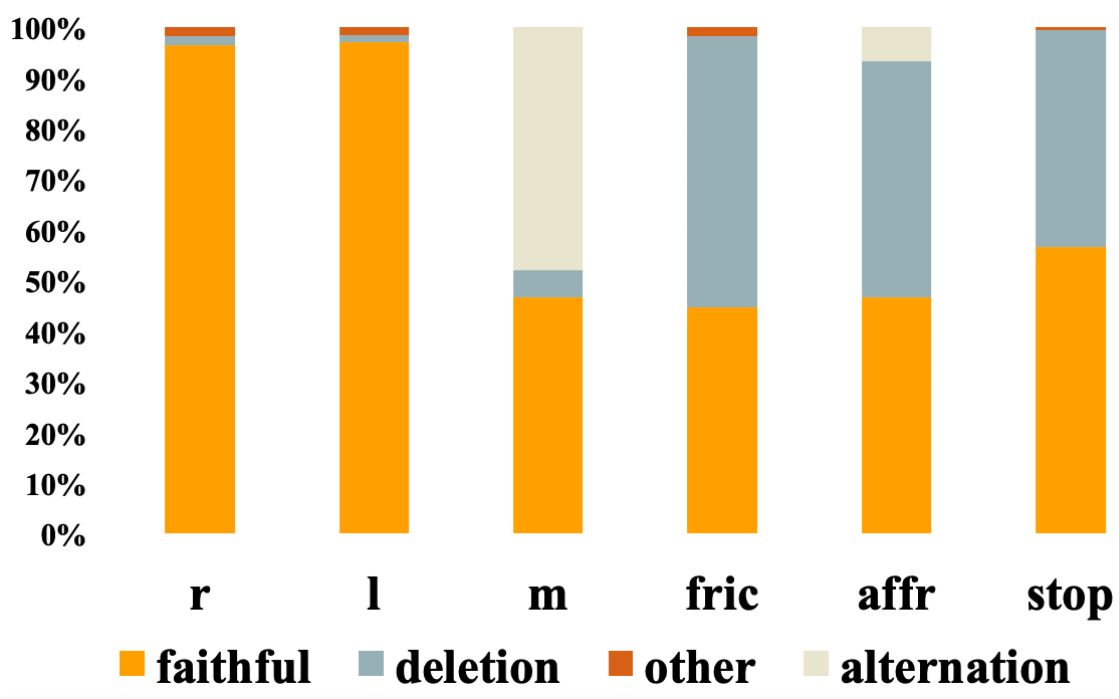

Figure 2: Adaptation of English codas

A sonority effect abstracted from the coda adaptation patterns in Figure 2 is that consonants with higher sonority are more likely to be faithfully produced in coda positions. This is consistent with not only the contrast between the sonorants / $1 /$ and obstruents, but also the fact that $/ \mathrm{m} /$ is partially preserved when it is changed to $/ \mathrm{n} /$ by the virtue of the faithfully produced nasal feature.

3.3 TONAL ADAPTATION. In native Mandarin phonotactics, each syllable has a tone. When Mandarin-speakers code-switch to English in the A-not-A construction, the declarative intonation of the English word, which is a falling intonation from the primary-stressed syllable to the end of the word, is preserved in the base. For example, the declarative intonations of believe, wash, and washable are shown in (4).

(4) declarative intonations of believe, wash, and washable
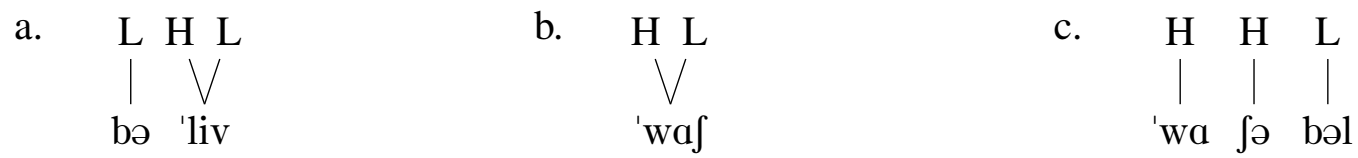

These three words represent three intonation patterns. In the three patterns of tonal adaptations in the reduplication process of A-not-A constructions in (5), the tone of the reduplicant is always faithful to the tone of the first syllable in the base.

(5) Three patterns of tonal adaptations: 

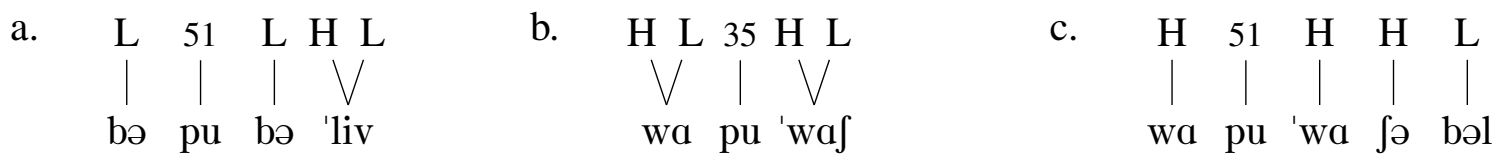

The tone of the base word not only affect the tones of the reduplicants, but also triggers the allomorph alternation of the Mandarin adverb $b u[\mathrm{pu}]$ which is linearly between the reduplicant and the base. In Mandarin, the allomorph alternation of $b u[\mathrm{pu}]$ is triggered by the following tone, as shown in (6).

Tone alternation of $b u[\mathrm{pu}]$ in Mandarin

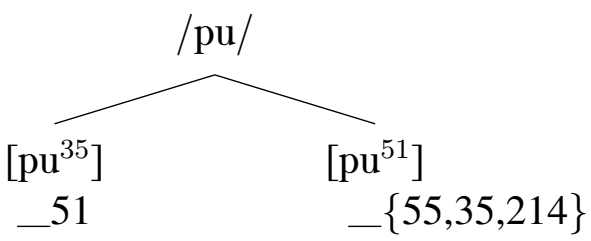

While the intonation of English words varies along the sentence purpose (e.g., declarative intonation, interrogative intonation, etc.), the English word borrowed as base in Mandarin A-notA constructions only bears the contour that is faithful to its declarative intonation in English, which indicates that the declarative intonation of the English input is internalized as the tone of the base word in the A-not-A context. This is why the tone of the base can trigger the allomorph alternation of the $b u[\mathrm{pu}]$ preceding it. When the first syllable in the base has a falling tone similar to the Mandarin T4 [51], [pu] is assigned a T2 [35] (see (7-a)), otherwise [pu] has the T4 [51] in Mandarin (see (7-b)).
a. $\quad \mathrm{pu}^{35} \mathrm{wad}^{\mathrm{HL}}$
b. $\mathrm{pu}^{51} \mathrm{wa}^{\mathrm{H}} \int ə \mathrm{~b} ə \mathrm{l}$

3.4 SUmmary. This section illustrates the strategies used in segmental adaptations of English onsets and codas in Mandarin A-not-A constructions and the patterns of tonal adaptations observed in Mandarin-English code-switching in A-not-A constructions.

The effects of sonority are found in both onset and coda positions. When reduplicating syllables with complex onsets, speakers either faithfully produce the cluster or reduplicates only the initial consonant and insert a vowel to make it a syllable, splitting the original consonant sequences. Due to the Sonority Sequencing Principle, consonant clusters with sonority dipping are more likely to split and undergo vowel insertion than clusters with rising sonority. In the coda position, the effect of sonority is that consonants with higher sonority are more likely to be preserved, wholly or partially, in coda positions.

The tonal adaptations of English exist in both the base and the reduplicant of the A-not-A constructions. The tone of the base comes from the declarative intonation of its English input, and the tone of the reduplicant is faithfully reduplicated from the tone of the first syllable of the base.

4. Modeling segmental adaptations. This section presents a Maximum Entropy Harmonic Grammar (MaxEnt; Goldwater \& Johnson 2003) model of the segmental adaptation results from the production experiment introduced in section 2. In the MaxEnt framework, a set of 
weighted constraints impose restrictions on possible patterns in the data and violations of multiple weighted constraints are summed together. Compared to classical Optimality Theory (OT; Prince \& Smolensky 2004), a MaxEnt model can better handle the variations observed in the experiments. The complete list of constraints in the model and their definition is shown in table 4.

$\begin{array}{ll}\mathrm{RED}=\sigma & \text { penalizes reduplicating more than one syllables } \\ \mathrm{ID}-\mathrm{V} & \text { penalizes any change in vowel features } \\ \mathrm{ID}-\mathrm{BR} & \text { penalizes any change in RED-BASE mappings } \\ \mathrm{DEP}-\mathrm{BR} & \text { penalizes epenthesized segments in RED } \\ \mathrm{MAX}-\mathrm{BR} & \text { penalizes deleted segments in RED } \\ * \mathrm{CCC}_{\mathrm{onset}} & \text { penalizes triple-consonant cluster in onset } \\ * \mathrm{SC}_{\text {onset }} & \text { penalizes an onset cluster with a consonant following [s] } \\ * \mathrm{Cl}_{\text {onset }} & \text { penalizes an onset cluster with a consonant preceding [1] } \\ * \mathrm{Cr}_{\text {onset }} & \text { penalizes an onset cluster with a consonant preceding [r] } \\ \mathrm{MAX}-[\mathrm{son}]_{\text {coda }} & \text { penalizes a [+son] feature in the coda position that is in } \\ & \text { the base but not in the reduplicant } \\ * \mathrm{~m}_{\text {coda }} & \text { penalizes coda [m] } \\ *[-\mathrm{son}]_{\text {coda }} & \text { penalizes obstruent coda } \\ *[+ \text { approx }]_{\text {coda }} & \text { penalizes approximate coda } \\ & \quad \text { Table 4: Constraint definitions }\end{array}$

The model was fitted with the observed frequencies of each A-not-A construction candidate in the experiment. The resulting constraint weights are shown in table 5.

\begin{tabular}{|c|c|c|c|c|c|}
\hline $\mathrm{RED}=\sigma$ & 3.79 & $*_{\mathrm{S}} \mathrm{C}_{\text {onset }}$ & 3.540 & MAX-[son $]_{\text {coda }}$ & 1.523 \\
\hline ID-V & 3.691 & $* \mathrm{CCC}_{\text {onset }}$ & 1.558 & $* \mathrm{~m}_{\text {coda }}$ & 1.493 \\
\hline ID-BR & 1.686 & $* \mathrm{Cl}_{\text {onset }}$ & 1.312 & $*[- \text { son }]_{\text {coda }}$ & 0.669 \\
\hline DEP-BR & 0.986 & $* \mathrm{Cr}_{\text {onset }}$ & 0 . & $*[+ \text { approx }]_{\text {coda }}$ & 0 . \\
\hline MAX-BR & 0.755 & & & & \\
\hline
\end{tabular}

Table 5: Constraint weights in the MaxEnt model $\left(r^{2}=0.769\right)$

5. Summary and Discussion. This paper investigates how Mandarin-speakers adapt English words to Mandarin phonotactics in the A-not-A constructions that reduplicate the first syllable of the base.

I conducted a production experiment with 55 English words and collected data from 17 native Mandarin-speakers. The strategies used in onset adaptations include faithful production and partial reduplication with vowel insertion. The strategies used in coda adaptations are faithful production, deletion, and alternation. The effects of sonority are found in both onset and coda positions in that complex onsets with sonority dipping are more likely to split and undergo vowel insertion than those with rising sonority, and that consonants with higher sonority are more likely to be preserved in coda positions. I also found that when Mandarinspeakers code-switch to English while producing A-not-A constructions, the tone of the English base comes from the declarative intonation of its English input,and the tone of the redu- 
plicant is copied from the first tone in the base. Finally, I modeled the experiment results with MaxEnt model and presented the model in section 4. With studies of this kind, we can identify those hidden constraints in Mandarin phonology that capture the sonority effects in both onset and coda positions.

Although code-switching to English is not uncommon in Mandarin colloquial speech, especially for speakers with great English exposure, the adaptations analyzed in this paper do not occur when the English words are simply used ad hoc in the speech, but occur only when the English words undergo some native Mandarin phonological process such as the reduplication in A-not-A constructions. With respect to the phonological restrictions, what the reduplication process generates in this study is between loan words and the English bases borrowed ad hoc in the A-not-A forms. Loan words in Mandarin are phonologized into it and obey its phonological rules as native words do. The English reduplicants in the A-not-A forms have to be adapted to Mandarin to some degree under the pressure of both base-reduplicant faithfulness and markedness constraints in Mandarin phonotactics. The bases borrowed in the A-not-A constructions are the product of code-switching, thus most faithful to the English input. An intriguing question to ask is why is a borrowed word only undergoes such adaptations when a phonological process such as reduplication applies to it but not as soon as it is borrowed?

\section{References}

Goldwater, Sharon \& Mark Johnson. 2003. Learning OT constraint rankings using a maximum entropy model. In Jennifer Spenader, Anders Eriksson \& Östen Dahl (eds.), Proceedings of the Stockholm Workshop on Variation within Optimality Theory. 111-120. Stockholm: Stockholm University Department of Linguistics.

Prince, Alan \& Paul Smolensky. 2004. Optimality theory: Constraint interaction in generative grammar. Oxford: Blackwell. 